\title{
Secondary Structure of an RNA Aptamer Binding to G-rich Sequence RNA
}

\author{
Sei-Young Choi and Bongrae Cho* \\ Department of Applied Chemistry, Cheongju University, Cheongju 360-764, Korea. ${ }^{*}$ E-mail: brcho@cju.ac.kr \\ Received June 27, 2011, Accepted August 9, 2011
}

Key Words : G-rich RNA, RNA aptamer, RNase T1, Nuclease S1, RNase V1

RNA structure has sometimes been analyzed by the biophysical method of NMR (nuclear magnetic resonance), which has not been popular because the RNA with small size only is available and a large amount of RNA is required for the structural analysis with NMR. ${ }^{1}$ So biochemical methods using structure specific enzymes and chemicals have widely been used for the analysis of RNA structure. ${ }^{2-10}$ Enzymes and chemicals which have mainly been used for probing RNA structure in solution, are as follows; doublestrand-specific RNase V1, single-strand-specific nuclease $\mathrm{S} 1$, RNase $\mathrm{T} 1$ that has the specificity for a guanine in single strand region, and DMS (dimethyl sulfate) that methylates position N-1 of adenines and, to a lower extent, N-3 of cytosines, CMCT (1-cyclohexyl-3-(2-morpholinoethyl)carbodiimide metho- $p$-toluene sulfonate) that modifies position N-3 of uridine and, to a weaker degree, N-1 of guanines and kethoxal (3-ethoxy-1,1-dihydroxy-2-butanone) that modifies position $\mathrm{N} 1$ and $\mathrm{N} 2$ of guanine in the single strand. Hydroxyl radical $(\cdot \mathrm{OH})$ has also been used for the high-order structure analysis of RNA. Exposed nucleotides are damaged by hydroxyl radical while nucleotides involved in tertiary contacts are protected from the damage, making it a favorable approach for establishing exterior/interior relations for RNA structure. ${ }^{11-17}$ Radicals are generated from $\mathrm{Fe}(\mathrm{II})$-EDTA with hydrogen peroxide $\left(\mathrm{H}_{2} \mathrm{O}_{2}\right)$. Ascorbate (or DTT) is added to reduce Fe(III) to Fe(II). Hydrogen abstraction from the ribose 4 ' carbon leads to strand scission. In-line probing is also an RNA-structure probing method developed by the Breaker's group. ${ }^{18-20}$ This method has been used to examine the secondary structure of RNAs and whether RNAs undergo structural rearrangements under the different incubation conditions. In-line probing takes advantage of the fact that the spontaneous cleavage of RNA is dependent on the local structure at each inter-nucleotide linkage. RNA degrades through a nucleophilic attack by the 2 ' oxygen on the adjacent phosphorus. Cleavage occurs efficiently when the attacking 2 ' oxygen, the phosphorus and the departing 5 ' oxygen of the phosphodiester linkage are in a linear configuration. Linkages in double strand region of a folded RNA show resistance to cleavage because it is difficult for the atoms to be held in an in-line configuration. However, if folding does not restrict its structure, linkages occasionally take on in-line geometry through random motion and therefore are subject to a spontaneous cleavage.

RNA aptamers capable of binding to the guanine-rich sequence RNA (5'-GGGAGGGGCGGGUCUGGG-3') in

\section{GGGAUCCGCAUGCAAGCUUACACUCGGCGU}

Figure 1. Sequence of the randomized region in RNA aptamer 1130-32. The consensus sequence is underlined in bold letters.

the 5'-UTR (untranslated region) of N-ras oncogene were selected from a random-sequence RNA library with RNARNA interaction (manuscript in preparation). The selected RNA aptamers can recognize the specific domain of RNA structure like a monoclonal antibody and be candidates of the anticancer agent at the genetic level. The determination of the structure of selected RNA aptamers is very important prior to getting the information for the interaction between an RNA aptamer and a ligand RNA used for selection. So in this work, the secondary structure of an RNA aptamer 1130-32, one of the selected RNA aptamers (Fig. 1) was predicted with the theoretical method, the CLC RNA workbench ver. 4.2 program accessed on the internet (www.clcbio.com) and biochemically examined with RNA structural probes such as RNase T1, RNase V1 and nuclease S1.

The structure of RNA aptamer 11-30-32 including the primer sequence was probed in binding buffer with RNase T1, RNase V1, and nuclease S1 (Fig. 2). G12, G26, G37 and G41 were strongly cleaved by RNase T1, suggesting that these guanines are thought to be in the single-stranded region of the secondary structure model of RNA aptamer 1130-32. Nucleotide G16 in the double-stranded region was strikingly digested by RNase T1, suggesting that the base pair G16:C23 cannot be stable by the presence of the adjacent loop of C17UUACA22 and exist in both the singlestranded and double-stranded region at the equilibrium state. And G27, G29, G31 and G32 were not or weakly cleaved by RNase T1 so these guanines are thought to be in the doublestranded region.

C6CGC9, G12, A15, U30GG32 and C35 were strongly cleaved by RNase V1 so these nucleotides are thought to be located in the double-stranded region. But U18UA20 which was thought to be in the single-stranded region, was strongly susceptible to RNase V1, suggesting that these nucleotides could be stacked or have the tertiary interaction of pseudoknot with the promising candidate of the sequence of C42AA44 through intramolecular interaction and be accessible to RNase V1 in solution. Nucleotide G12 in the double-stranded region of the secondary structure model was strikingly digested by both RNase T1 and RNase V1, 


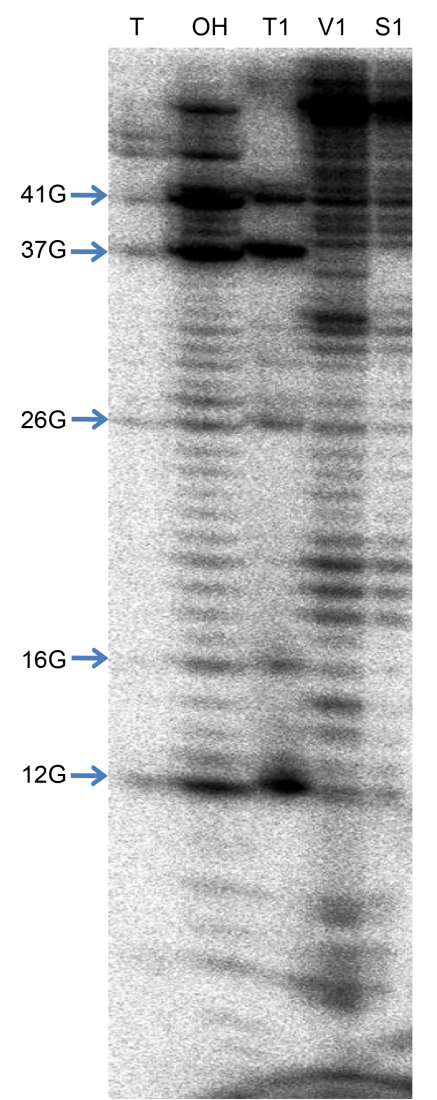

Figure 2. Enzymatic probing of RNA aptamer 11-30-32 labeled at the 5'-end. The RNA was partially digested with RNase T1, RNase $\mathrm{V} 1$ and nuclease $\mathrm{S} 1$. The cleaved nucleotides by RNase $\mathrm{T} 1$ are indicated by arrows. Lane T, the denatured RNA treated with RNase $\mathrm{T}$; ; lane $\mathrm{OH}$, partial alkaline ladder.

suggesting that the base pair of G12:C28 is not stable by the presence of a bulge A10U11 and can exist in both the singlestranded and double-stranded region at the equilibrium state.

Sequences U18UA20 and C38AUG41 were strongly cleaved by nuclease $\mathrm{S} 1$ so these nucleotides are thought to be in the single-stranded region of the secondary structure model of RNA aptamer 11-30-32.

There was some difference in the secondary structure model of RNA aptamer 11-30-32 between thermodynamically predicted on the basis of base pairing and experimentally resulted with the structure-specific enzymes because the thermodynamical method has the limitation not to regard the tertiary interactions in RNA structure. The accurate secondary structure model is thought to be expected if we have the information for the tertiary interactions in RNA structure.

In conclusion, the structure of RNA aptamer 11-30-32 was analyzed in solution with the probes such as RNase T1, RNase V1, and nuclease S1 and its possible secondary structure was represented (Fig. 3). The results suggested that 1) this molecule has two single-stranded regions and one double-stranded region with a bulge A10U11, 2) the sequence of U18UA20 can be stacked or have the tertiary interaction of pseudoknot through intramolecular interaction and 3) the base pair of G12:C28 is not stable because of the adjacent

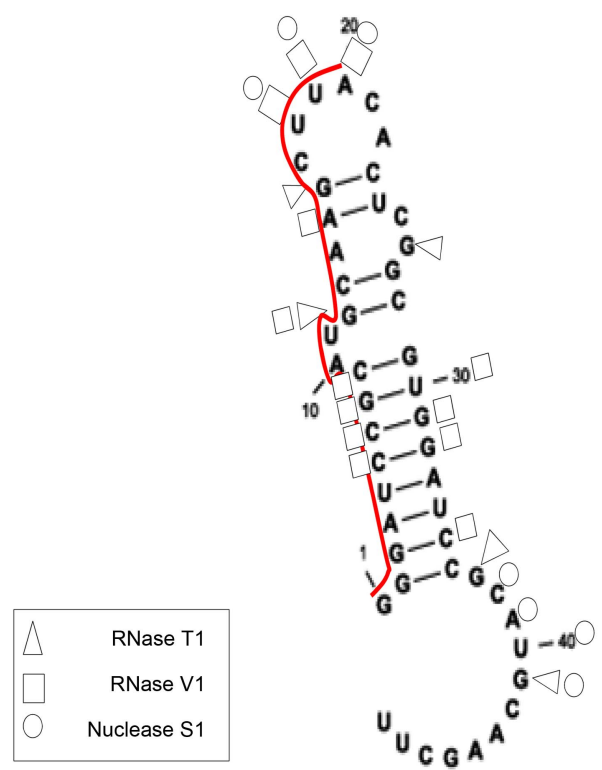

Figure 3. Schematic representation of possible secondary structure of RNA aptamer 11-30-32. Triangles indicate the sites cleaved by RNase T1, squares indicate the sites cleaved by RNase V1 and circles indicate the sites cleaved by nuclease $\mathrm{S} 1$.

presence of the single-stranded region.

\section{Experimental Section}

Preparation of RNA. RNA aptamer 11-30-32 was synthesized by run-off in vitro transcription with T7 RNA polymerase from the DNA template to which the T7 promoter was annealed and purified by gel elution of the crush and soak method. ${ }^{21}$ The resulting RNA was treated with CIP (calf intestinal alkaline phosphatase) to remove 5 ' terminal phosphate and then labeled at the $5^{\prime}$ end using $\left[\gamma_{-}{ }^{32} \mathrm{P}\right] \mathrm{ATP}$ and T4 polynucleotide kinase.

Enzymatic Cleavage Reaction. 5'-terminal radiolabeled RNA aptamer 11-30-32 was heated in binding buffer (30 $\mathrm{mM}$ Tris-acetate, $\mathrm{pH}$ 7.5, $60 \mathrm{mM}$ magnesium acetate, 120 $\mathrm{mM}$ potassium acetate, and $120 \mathrm{mM}$ ammonium acetate) at $90{ }^{\circ} \mathrm{C}$ for $2 \mathrm{~min}$ and allowed to cool to RT $\left(\sim 21{ }^{\circ} \mathrm{C}\right)$. Then 0.1-1 unit of nuclease S1 (Boehringer Manheim $\mathrm{GmbH}, \mathrm{W} .-$ Germany) or 0.001-0.01 unit of RNase V1 (Pierce Molecular Biology, Perbio) or 0.1-1 unit of RNase T1 (Industrial Research Limited) was added to the above mixture and then the reaction mixture was incubated for $25 \mathrm{~min}$ at RT. The reaction volume included an additional $1 \mathrm{mM} \mathrm{ZnCl}_{2}$ for nuclease $\mathrm{S} 1$ cleavage. The cleavage products were recovered by ethanol precipitation and separated on a $15 \%$ polyacylamide gel in $90 \mathrm{mM}$ Tris-borate $(\mathrm{pH} 8.3)$ and $2.5 \mathrm{mM}$ EDTA containing $7 \mathrm{M}$ urea.

\section{References}

1. Lee, J.-H. Bull. Korean Chem. Soc. 2008, 29, 1937.

2. Ko, J.-H.; Cho, B.; Ahn, J. K.; Lee, Y.; Park, I. Bull. Korean Chem. Soc. 1999, 20, 1335. 
3. Brunel, C.; Romby, P. Methods in Enzymology 2000, 318, 3.

4. Ko, J.; Lee, Y.; Park, I.; Cho, B. FEBS Lett. 2001, 508, 300.

5. Cho, B. Bull. Korean Chem. Soc. 2006, 27, 786

6. Cho, B. Bull. Korean Chem. Soc. 2007, 28, 689.

7. Cho, B. Bull. Korean Chem. Soc. 2008, 29, 1063.

8. Cho, B. Bull. Korean Chem. Soc. 2008, 29, 2026.

9. Cho, B. Bull. Korean Chem. Soc. 2009, 30, 1193.

10. Cho, B. Bull. Korean Chem. Soc. 2011, 32, 2137.

11. Latham, J.; Cech, T. Science 1989, 45, 276.

12. Stern, S.; Moazed, D.; Noller, H. Methods Enzymol. 1988, 164, 481.

13. Murphy, F. L.; Cech, T. R. Biochemistry 1993, 32, 5291.

14. Rangan, P.; Masquida, B.; Westhof, E.; Woodson, S. Proc. Natl.
Acad. Sci. USA 2003, 100, 1574.

15. Tsai, H.; Masquida, B.; Biswas, R.; Westhof, E.; Gopalan, V. J. Mol. Biol. 2003, 325, 661.

16. Barrera, A.; Pan, T. RNA 2004, 10, 482.

17. Bergman, N.; Lau, N.; Lehnert, V.; Westhof, E.; Bartel, D. P. RNA 2004, 10, 176

18. Soukup, G. A.; Breaker, R. R. RNA 1999, 5, 1308.

19. Li, Y.; Breaker, R. R. J. Am. Chem. Soc. 1999, 121, 5364.

20. Mandal, M.; Breaker, R. R. Mol. Cell Biol. 2004, 5, 451.

21. Sambrook, J.; Fritsch, E. F.; Maniatis, T. 1989, Molecular Clong: A Laboratory Mannual, 2nd ed.; Cold Spring Harbor Laboratory Press: Cold Spring Harbor, NY. 